\title{
Research on Design Parameters and Fatigue Life of Tunnel Bottom Structure of Single-Track Ballasted Heavy-Haul Railway Tunnel with 40-Ton Axle Load
}

\author{
W. B. Ma, ${ }^{1,2}$ J. F. Chai ${ }^{1,}{ }^{1,2}$ Z. L. Han, ${ }^{1,2}$ Z. G. Ma, ${ }^{1,2}$ X. X. Guo, ${ }^{1,2}$ W. H. Zou, ${ }^{1,2}$ Z. L. An, ${ }^{1,2}$ \\ T. F. Li, ${ }^{1,2}$ and Y. B. Niu ${ }^{1,2}$ \\ ${ }^{1}$ Railway Engineering Research Institute, China Academy of Railway Sciences Corporation Limited, Beijing 100081, China \\ ${ }^{2}$ State Key Laboratory for Track Technology of High-speed Railway, Beijing 100081, China \\ Correspondence should be addressed to J. F. Chai; chaijinfei@rails.cn
}

Received 23 August 2020; Revised 5 October 2020; Accepted 7 November 2020; Published 21 November 2020

Academic Editor: Zheng-zheng Wang

Copyright (c) 2020 W. B. Ma et al. This is an open access article distributed under the Creative Commons Attribution License, which permits unrestricted use, distribution, and reproduction in any medium, provided the original work is properly cited.

\begin{abstract}
The coupling calculation model of tunnel and surrounding rock is established by the finite difference method, and the main design parameters of lining structure of single-track ballasted tunnel under 40-ton axle load, heavy train load, are studied in combination with cumulative damage mechanism of surrounding rock at tunnel bottom and the fatigue life characteristics of concrete structure at tunnel bottom. The results show that (1) inverted arch shall be set in sections of III-grade and above. Whether an invert is set in sections of II-grade and below shall be determined according to lithology and groundwater conditions. When the surrounding rock condition is good and the tunnel bottom structure (without invert structure) is adopted, the thickness is recommended to be more than $20 \mathrm{~cm}$, and the concrete strength grade should not be lower than C35. (2) Connection mode: the inverted arch and side wall of the tunnel should be connected in sequence to reduce the stress concentration and improve the stress state of the connection part between the inverted arch and the side wall. (3) It is suggested that the rise-span ratio of invert of single-track tunnel should be $1 / 6 \sim 1 / 8$; the larger value should be taken when the surrounding rock condition is poor and the small value should be taken when the surrounding rock condition is good. (4) The thickness of inverted arch is recommended to be no less than $20 \mathrm{~cm}$ under the condition of V-grade surrounding rock, to be no less than $15 \mathrm{~cm}$ under IV-grade surrounding rock, and to be no less than $10 \mathrm{~cm}$ under the condition of III-grade surrounding rock and II-grade surrounding rock sections requiring inverted arch. (5) The recommended value of bedding thickness meeting the design service life is $20 \mathrm{~cm}$ under the condition of II-grade surrounding rock.
\end{abstract}

\section{Introduction}

Heavy-haul railway has become the best choice for transporting bulk goods in the world because of its advantages of large volume, high speed, low energy consumption, and low cost. A lot of researchers have studied the heavy-haul railway and achieved a series of results.

Jakobsen obtained the relationship between ground vibration level and relevant track parameters through field test of ground vibration caused by train load [1]. Dawn et al. tested the vibration response of British railway subgrade and studied the relationship between train speed, excitation frequency, and track parameters [2]. Based on a heavy-haul railway tunnel project, Zou et al. studied the stress distribution and dynamic response of the foundation structure of heavy-haul railway tunnel under the action of train load of 30-ton axle load combined with field vehicle test and numerical simulation [3]. Li et al. clarified the dynamic characteristics of ballasted and ballastless double-track heavy-haul railway tunnel structure under the conditions of $27 \mathrm{t} \sim 30 \mathrm{t}$ axle load and III $\sim \mathrm{V}$ surrounding rock through field measurement [4]. Chang studied the influence of key 
parameters on the dynamic response of heavy-haul track structure with 30-ton axle load and provided suggestions for the selection of structural parameters by analyzing the static and dynamic characteristics of heavy-haul track structure [5]. Huo simulated and analyzed the fatigue damage of heavy-haul lines and concluded that the track bed, invert filling layer, and invert of tunnel bottom structure all showed damage in turn [6]. Liu et al. carried out wave fatigue test of double-side high limit static load and low dynamic load according to the mechanical characteristics of key fatigue parts at the bottom of heavy-haul railway tunnel and obtained the evolution law of maximum tensile strain and electrical conductivity of structural specimens at the bottom of heavy-haul railway tunnel [7]. Song analyzed and obtained the influence rules of parameters such as train speed, width of track bed slab, thickness of track bed slab, stiffness of tunnel foundation, and other parameters on dynamic performance of heavy-haul vehicle ballastless track system and proposed reasonable value selection suggestions for structural parameters of ballastless track in heavy-haul railway tunnel [8]. Li researched the energy evolution characteristics of hard rock during triaxial failure with different loading and unloading paths and the Brazilian disc test for rock mechanics applications [9-11]. Wang researched the shaking table tests of tunnel linings in progressive states of damage and analyzed the seismic damage classification and risk assessment of mountain tunnels [12-16]. Chai studied the internal fracture mechanism of simulated rock acoustic emission events using T-k diagram method and P-T graph method [17-19]. Cheng researched the element-free Galerkin method based on the nonsingular weight functions [20-23].

At present, the "Heavy-Haul Railway Design Code" (TB10625-2017) of China is usually applicable to heavy-haul railway with axle loads less than 30 tons. There is still insufficient understanding of the stress distribution characteristics and dynamic response of railway tunnel structure with 40-ton axle load. Therefore, it is inevitable that railway tunnels with 40-ton axle load under construction and completed will inevitably have greater hidden dangers due to the bearing of heavy train load on the foundation structure.

In this paper, the coupling calculation model of tunnel and surrounding rock is established by the finite difference method, and the main design parameters of lining structure of single-track ballasted tunnel under 40-ton axle load heavy train load are studied in combination with cumulative damage mechanism of surrounding rock at tunnel bottom and the fatigue life characteristics of concrete structure at tunnel bottom. This study has important reference significance for the design parameters of heavy-haul railway tunnel lining with 40-ton axle load.

\section{Dynamic Performance Analysis of Tunnel Bottom Structure}

The finite difference method is used to establish a dynamic numerical analysis model to calculate the dynamic response characteristics of the foundation structure of heavy-haul railway tunnel under the action of train load.

\subsection{Numerical Calculation Model}

2.1.1. Numerical Computation Grid. The numerical model grid under typical working conditions is shown in Figure 1. The model size is $60 \mathrm{~m} * 12 \mathrm{~m} * 60 \mathrm{~m}$. The unit thickness is taken along the tunnel axis direction, the width is taken as $30 \mathrm{~m}$ from the tunnel axis to both sides, and the distance between the upper and lower boundaries in the height direction and the tunnel axis is $30 \mathrm{~m}$.

2.1.2. Calculation Condition. The tunnel section in BFS stage is taken as the typical calculation section. The influence of different design parameters on dynamic response and damage characteristics of tunnel foundation structure is mainly aimed at. The calculation conditions are shown in Table 1.

The benchmark example of the above conditions is as follows: the axle load of the train is 40 tons, the surrounding rock grade is grade IV, the invert thickness is $20 \mathrm{~cm}$, and the rise-span ratio is $1 / 6$. Other conditions only need to change the corresponding parameters.

2.1.3. Calculation Parameters. The physical and mechanical parameters of surrounding rock are selected according to the survey and design data of typical tunnels and referring to similar strata. The basic mechanical parameters of invert, filling layer, primary support, secondary lining, and other tunnel structural materials are selected according to the code for "Design of Railway Tunnel" (TB 10003-2005), and equivalent treatment is conducted for anchor bolt and reinforcement. See Table 2 for specific parameters.

2.1.4. Simulation Method of Train Dynamic Load. The factors that should be considered in the simulation of train dynamic load include axle load, suspension mass, running speed, and line smoothness. The commonly used simulation methods are field test method, artificial simulation excitation method, and train track coupling model method. The field test method is affected by the load, speed, track state, and other factors, so the test results obtained by the field test method are more discrete, and it is difficult to accurately describe with the expression and can only analyze the specific working conditions; the train track coupling model connects the train model and the track model through the assumed wheel rail contact relationship, which is more comprehensive, but the model and parameters are too complex, and the wheel rail contact relationship is not. The artificial simulation excitation method uses an excitation function fitted by empirical formula to simulate train load, which can fully consider the influence of axle load, speed, and other factors, and its form is relatively simple and widely used at present.

In this paper, the excitation force function is used to simulate the heavy-haul train load. The excitation force function includes the static load and the vibration load reflecting the factors such as irregularity and rail surface waveform wear effect. The superposition combination of train wheel set force and the scattered transmission of rail and sleeper is considered. 


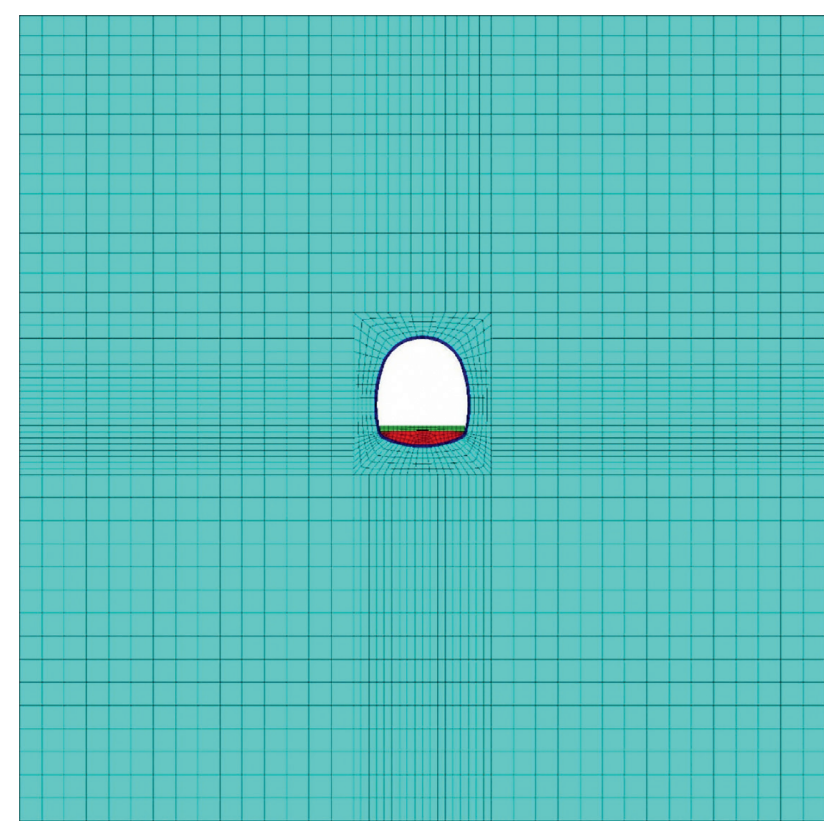

Figure 1: Numerical calculation model.

TABLE 1: Calculation conditions under different design parameters of tunnel foundation structure.

\begin{tabular}{lccc}
\hline $\begin{array}{l}\text { Influence } \\
\text { factor }\end{array}$ & $\begin{array}{c}\text { Grade of } \\
\text { surrounding rock }\end{array}$ & $\begin{array}{c}\text { Invert/floor } \\
\text { thickness }(\mathrm{cm})\end{array}$ & $\begin{array}{c}\text { Invert rise- } \\
\text { span ratio }\end{array}$ \\
\hline \multirow{3}{*}{ Specific } & II & 10 & $1 / 5$ \\
parameters & III & 15 & $1 / 6$ \\
& IV & 20 & $1 / 7$ \\
& V & 30 & $1 / 8$ \\
\hline
\end{tabular}

$$
F(t)=k_{1} k_{2}\left(P_{0}+P_{1} \sin \omega_{1} t+P_{2} \sin \omega_{2} t+P_{3} \sin \omega_{3} t\right)
$$

where $P_{0}$ is the static load of the wheel; $P_{1}, P_{2}, P_{3}$ are the typical vibration load values related to the ride irregularity, dynamic additional load, and waveform wear, respectively, corresponding to a typical value in the control conditions (1)-(3) in Table 3; $k_{1}$ is the wheel rail force superposition coefficient; and $k_{2}$ is the rail dispersion transmission coefficient.

The amplitude of vibration load is

$$
P_{i}=m a_{i} \omega_{i}, \quad i=1,2,3,
$$

where $m$ is the unsprung mass of the train; $a_{i}$ is the typical rise height, corresponding to the situations of (1), (2), and (3) in Table $3 ; \omega_{i}$ is the vibration circumference ratio under the condition of irregularity control, and the expression is $\omega_{i}=2 \pi v / L_{i}, v$ is the running speed, and $L_{i}$ is the wavelength management value of track geometric irregularity, corresponding to (1), (2), and (3) in Table 3.

\subsubsection{Boundary Conditions of Dynamic Analysis and Ma- terial Damping Model}

1. Artificial Boundary Conditions. The setting of artificial boundary conditions and the input of vibration wave are the key links to realize the dynamic numerical analysis, which directly affects the accuracy and credibility of the calculation results. In the engineering rock mass of semi-infinite space, when the vibration wave propagates around, it will transmit and refract when it meets the structure or rock structure plane and will interfere with the incident wave to form a complex wave field. In the near-field dynamic numerical analysis, in order to effectively simulate the scattering wave passing through the truncated boundary of the model and make the vibration wave energy dissipate to infinity, it is necessary to introduce artificial boundary conditions to eliminate the reflection and oscillation of the vibration wave on the artificially cut boundary in the calculation area. In the FLAC3D dynamic analysis of this chapter, the free field boundary is used as the dynamic analysis boundary condition.

In the numerical simulation of vibration response of near-surface structures such as slopes and shallow underground structures, the lateral boundary of the numerical model must consider the free field motion without structures. In the numerical simulation, the free field motion is applied to the model boundary to keep the artificial boundary non-reflective. The lateral boundary of the discrete model is coupled with the free field to simulate the viscous boundary. The unbalanced force on a surface parallel to the axis in the normal direction can be expressed as follows:

$$
\left\{\begin{array}{l}
F_{x}=-\rho C_{p}\left(v_{x}^{m}-v_{x}^{f f}\right) A+F_{x}^{f f}, \\
F_{y}=-\rho C_{s}\left(v_{y}^{m}-v_{y}^{f f}\right) A+F_{y}^{f f}, \\
F_{z}=-\rho C_{s}\left(v_{z}^{m}-v_{z}^{f f}\right) A+F_{z}^{f f},
\end{array}\right.
$$

where $\rho$ is the density of material medium; $C_{p}$ and $C_{s}$ are the wave velocity of $\mathrm{P}$-wave and $\mathrm{S}$-wave in the material medium; $A$ is the influence area of free field node; $v_{x}^{m}, v_{y}^{m}, v_{z}^{m}, v_{x}^{f f}, v_{y}^{f f}$, $v_{z}^{f f}$ represent the three components of particle vibration velocity along the coordinate axis on the main node and corresponding free field node on the artificial boundary, respectively; and $F_{x}^{f f}, F_{y}^{f f}$, and $F_{z}^{f f}$ are the contribution of normal stress and tangential stress of node influence element in free field.

If the calculation model is a homogeneous medium without near-surface structure, the motion law of the calculation area is consistent with that of the free field, and the lateral damper does not work; when the two move relatively, the damper absorbs the energy of the traveling wave. In FLAC3D, the free field boundary conditions require that the bottom of the model is horizontal, and the normal direction is the axis; the side must be vertical, and the normal direction is $x$-axis or $y$-axis.

2. Material Damping Model. Material damping is a professional term to describe the energy dissipation mode of structural materials in the process of vibration. It means the energy dissipation of materials under the action of external dynamic load due to the friction between molecules in the medium, the sliding between structural planes, and the plastic response of materials. According to the different mechanism of damping, the damping forms in dynamic 
TABLE 2: The physical and mechanical parameters of materials.

\begin{tabular}{|c|c|c|c|c|c|}
\hline Structure & Elastic modulus (GPa) & Poisson's ratio & Density $\left(\mathrm{kN} \cdot \mathrm{m}^{-3}\right)$ & Cohesion $(\mathrm{kPa})$ & Friction angle $\left({ }^{\circ}\right)$ \\
\hline Ballast & 32.0 & 0.18 & 25.0 & & \\
\hline Filling layer & 29.0 & 0.18 & 25.0 & & \\
\hline Invert & 30.0 & 0.18 & 25.0 & & \\
\hline Second lining & 30.0 & 0.18 & 25.0 & & \\
\hline Primary lining & 27.5 & 0.18 & 25.0 & & \\
\hline Surrounding rock & 1.5 & 0.36 & 19.5 & 200 & 42 \\
\hline
\end{tabular}

TABLE 3: Simulation analysis parameters of track geometric irregularity.

\begin{tabular}{lcc}
\hline Control conditions & $\begin{array}{c}\text { Wavelength } \\
(\mathrm{m})\end{array}$ & $\begin{array}{c}\text { Sagittal } \\
(\mathrm{mm})\end{array}$ \\
\hline & 50.00 & 16.00 \\
According to ride comfort(1) & 20.00 & 9.00 \\
& 10.00 & 5.00 \\
According to the dynamic additional & 5.00 & 2.50 \\
load acting on the line(2) & 2.00 & 0.60 \\
& 1.00 & 0.30 \\
Waveform wear(3) & 0.50 & 0.10 \\
& 0.05 & 0.005 \\
\hline
\end{tabular}

analysis are divided into internal damping, external friction damping, sliding friction damping, plastic damping, and spatial dissipation damping. Different damping forms have different calculation methods. Rayleigh damping is used as material damping model in FLAC3D dynamic analysis in this chapter.

Rayleigh damping was first applied to the dynamic analysis of structures and elastic medium systems under natural vibration modes.

$$
C=\alpha M+\beta K
$$

where $\alpha$ is the proportional damping constant of the mass matrix $M$ and $\beta$ is the proportional damping constant of the stiffness matrix $K$. For a multi-degree-of-freedom system, the relationship between the damping constant and the damping ratio $\xi_{i}$ and the system frequency $\omega_{i}$ of the corresponding mode is as follows:

$$
\xi_{i}=\frac{\left(\left(\alpha / \omega_{i}\right)+\beta \omega_{i}\right)}{2}
$$

It can be seen from equation (5) that the damping ratio tends to increase rapidly when the frequency is very high or low. In contrast, the mass proportional damping has a greater contribution to the damping of the system in the low frequency band, while the stiffness proportional damping has a dominant contribution in the high frequency band. In the fundamental frequency band, the damping effect of mass term is equivalent to that of stiffness term:

$$
\begin{aligned}
& \alpha=\xi_{\text {min }} \omega_{\text {min }}, \\
& \beta=\frac{\xi_{\text {min }}}{\omega_{\text {min }}},
\end{aligned}
$$

where $\xi_{\min }$ is the minimum damping ratio and $\omega_{\min }$ is the system reference frequency. The center frequency $f_{\min }$ is defined as

$$
f_{\min }=\frac{\omega_{\min }}{2 \pi}
$$

and when the damping ratio $\xi_{\min }$ and center frequency $f_{\min }$ are known, the Rayleigh damping constants can be obtained. In FLAC3D explicit time domain dynamic calculation, the stiffness damping term in Rayleigh damping model often reduces the critical time step. For geotechnical materials, the damping ratio is usually desirable; for structural systems, the damping ratio is usually desirable; when the material in the stress system adopts the inelastic constitutive model, the plastic deformation will dissipate most of the energy, so for large deformation dynamic analysis, the damping ratio is usually smaller.

2.1.6. Numerical Simulation Steps. The specific steps of numerical simulation are as follows:

(1) The model grid is established, the rock mass parameters are given, the static boundary conditions are applied, and then the initial in situ stress field is generated.

(2) Simulation of excavation process. After the full face excavation, the in situ stress will be released by $10 \%$ and $20 \%$ after the initial support. The remaining in situ stress will be released after the secondary lining construction, and then the filling layer and ballast bed will be constructed.

(3) The dynamic response of the tunnel structure is obtained by applying normal and tangential dynamic boundary conditions to the boundary and applying heavy load train load in the form of artificial excitation force.

2.2. Analysis of Simulation Results. The dynamic response of the foundation structure of heavy-haul railway tunnel under the conditions of different surrounding rock grade, different invert thickness, and different invert risespan ratio is analyzed. The stress and dynamic response analysis is carried out at the top of filling layer, the top and bottom of inverted arch, the bottom of side wall arch foot, and the bottom of $1 / 4$ position of inverted arch. See Figure 2.

The vertical dynamic stress of measuring points 1,2 , and 3 under different working conditions is monitored to master the influence of design parameters of tunnel foundation structure on the vertical transmission law of train load. 


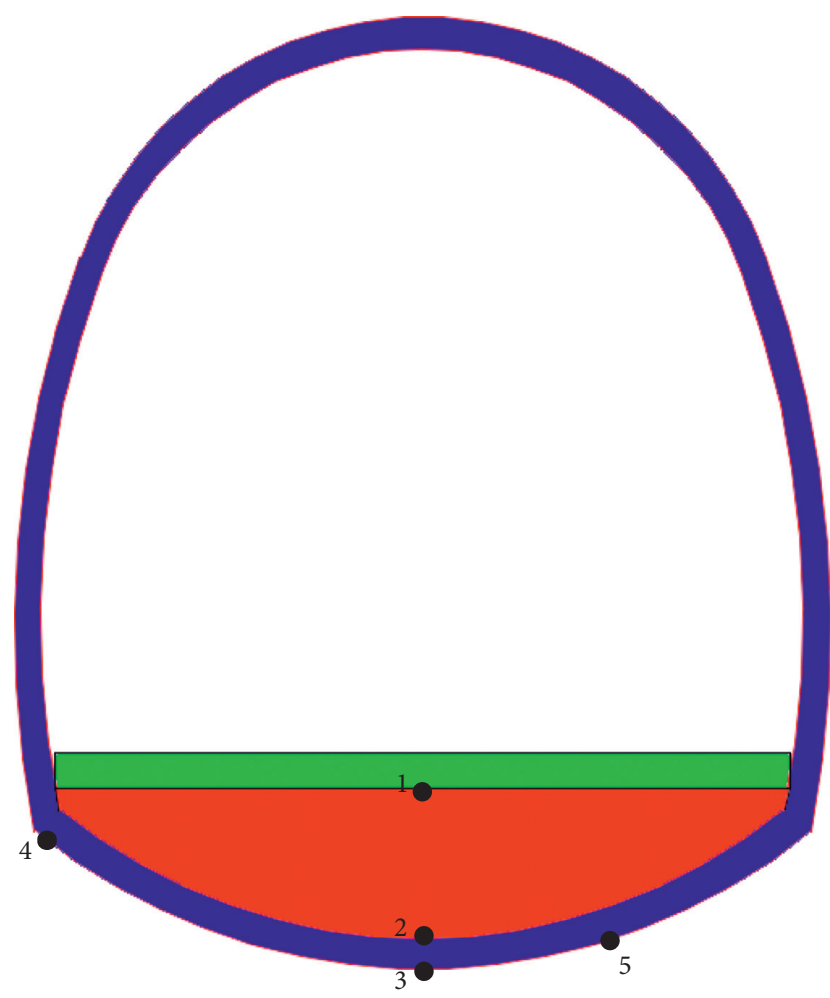

FIgURE 2: Layout of measuring points.

2.2.1. Influence of Surrounding Rock Grade. The vertical dynamic stress amplitude of measuring points 1, 2, and 3 under different surrounding rock levels can be seen in Table 4 . The vertical dynamic stress amplitudes of point 1 are close to each other under different surrounding rock grades; the vertical dynamic stress amplitudes of measuring points 2 and 3 decrease with the increase of surrounding rock grade, and the amplitude of measuring point 3 decreases more.

2.2.2. Influence of Invert Thickness. The vertical dynamic stress time history amplitudes of measuring points 1, 2, and 3 with different invert thickness are shown in Table 5. The results show that the vertical dynamic stress amplitude of measuring point 1 is close to that of measuring point 1 under different invert thickness conditions; the vertical dynamic stress amplitude of measuring point 2 increases with the increase of invert thickness; the vertical dynamic stress amplitude of measuring point 3 decreases with the increase of invert thickness.

2.2.3. Influence of Invert Rise-Span Ratio. The vertical dynamic stress amplitude of measuring points 1,2, and 3 under different rise-span ratio can be seen in Table 6 . The vertical dynamic stress amplitudes of point 1 are close to each other under different invert rise-span ratios, while the vertical dynamic stress amplitudes of measuring point 2 and measuring point 3 decrease with the increase of invert rise-span ratio. The dynamic response of heavy-haul railway tunnel foundation structure under different base structure design
TABLE 4: The vertical dynamic stress amplitude of measuring points 1,2 , and 3 under different surrounding rock grades.

\begin{tabular}{lcccc}
\hline \multirow{2}{*}{ Measuring point } & \multicolumn{4}{c}{ The vertical dynamic stress amplitude of } \\
& \multicolumn{2}{c}{ different surrounding rock grades $(\mathrm{kPa})$} \\
& II & III & IV & V \\
\hline 1 & 163.324 & 161.196 & 162.393 & 161.728 \\
2 & 113.449 & 96.558 & 88.844 & 79.667 \\
3 & 93.765 & 66.899 & 52.402 & 35.777 \\
\hline
\end{tabular}

TABLE 5: The vertical dynamic stress amplitude of measuring points 1,2 , and 3 under different invert thickness conditions.

\begin{tabular}{lcccc}
\hline \multirow{2}{*}{ Measuring point } & \multicolumn{4}{c}{ Vertical dynamic stress amplitude of } \\
& different invert thickness conditions $(\mathrm{kPa})$ \\
& $10 \mathrm{~cm}$ & $15 \mathrm{~cm}$ & $20 \mathrm{~cm}$ & $30 \mathrm{~cm}$ \\
\hline 1 & 162.127 & 162.393 & 162.393 & 161.595 \\
2 & 80.864 & 86.317 & 88.844 & 92.036 \\
3 & 59.584 & 56.126 & 52.402 & 48.545 \\
\hline
\end{tabular}

TABLE 6: The vertical dynamic stress amplitude of measuring points 1,2 , and 3 under different invert rise-span ratios.

\begin{tabular}{lcccc}
\hline \multirow{2}{*}{ Measuring point } & \multicolumn{4}{c}{ Vertical dynamic stress amplitude of } \\
& \multicolumn{4}{c}{ different invert rise-span ratios $(\mathrm{kPa})$} \\
& $1 / 8$ & $1 / 7$ & $1 / 6$ & $1 / 5$ \\
\hline 1 & 161.994 & 161.861 & 162.393 & 161.196 \\
2 & 96.691 & 92.834 & 88.844 & 82.593 \\
3 & 58.387 & 56.392 & 52.402 & 45.885 \\
\hline
\end{tabular}

parameters is analyzed numerically. The main conclusions are as follows.

(1) The thicker invert is helpful to improve the stress state of the tunnel base structure. The larger the thickness of the invert, the smaller the vertical dynamic stress at the bottom of the invert, but the range of the dynamic stress changes with it, and the less the influence of train dynamic load on the surrounding rock, which indicates that the thicker inverted arch is beneficial to the long-term stress stability of the tunnel bottom stratum.

(2) Under the condition of large invert span ratio (1/5), the smaller the vertical dynamic stress at the bottom of tunnel invert is $(45.885 \mathrm{kPa})$, the better improved the internal force of inverted arch structure can be, which can reduce the requirements of structural thickness and reinforcement to a certain extent and improve the stability and safety of the structure. But on the other hand, a large rise-span ratio, corresponding to thick invert filling, will increase the project cost to a certain extent.

(3) The worse the surrounding rock grade is, the more obvious the vertical dynamic stress attenuation along the base structure is; the greater the invert thickness is, the lower the load transfer rate at the bottom of inverted arch is; and the greater the invert rise-span ratio is, the lower the load transfer rate at the bottom of inverted arch is. 


\section{Design Parameters of Heavy-Haul Railway Tunnel Bottom with 40-Ton Axle Load}

3.1. Fatigue Life Prediction Method of Tunnel Bottom Structure. In the range of random fatigue load and high cycle fatigue zone, the Miner linear fatigue cumulative damage criterion can accurately meet the engineering requirements. So, Miner linear fatigue cumulative damage theory is generally used to analyze the fatigue life of the bottom structure of heavy-haul railway tunnel.

In the calculation of structural fatigue life, it is necessary to define the S-N curve of materials. Here, S-N curve refers to the relationship curve between stress amplitude and fatigue life. The calculation formula of stress amplitude is as follows:

$$
\sigma_{a}=\frac{\sigma_{\max }+\sigma_{\min }}{2}
$$

where $\sigma_{a}$ is the stress amplitude; $\sigma_{\max }$ is the maximum stress value in the cycle; and $\sigma_{\min }$ is the minimum stress value in the cycle.

Considering the maximum stress level and the minimum stress level, the $\mathrm{S}-\mathrm{N}$ fatigue life curve of concrete material is expressed as follows.

$$
\left\{\begin{array}{l}
\lg N=16.67-16.67 S_{t \max }+5.17 S_{t \max } \\
S_{\max }=\frac{\sigma_{\max }}{f} \\
S_{\min }=\frac{\sigma_{\min }}{f}
\end{array}\right.
$$

where $S_{\max }$ is the maximum stress level; $S_{\min }$ is the minimum stress level; and $f$ is the ultimate strength of the material.

The corresponding relationship between different stress amplitude and fatigue life can be obtained by formula (9). In addition, the influence of average stress on fatigue life is considered. The greater the average stress is, the smaller the fatigue life of the structure under each stress amplitude is. The average stress is expressed as follows:

$$
\sigma_{m}=\frac{\sigma_{\max }-\sigma_{\min }}{2}
$$

where $\sigma_{m}$ is the average stress.

According to the above formulas, the fatigue life of the tunnel bottom structure under various working conditions can be obtained. In the fatigue calculation, if the single intersection of a pair of unit 10000-ton trains in the tunnel is considered, the service life of the structure is

$$
T=\frac{N}{365 \times a},
$$

where $T$ is the service life of the structure; $N$ is the fatigue life, which is generally used to express $\lg N$; and $a$ is the number of 10000 -ton trains per day, with the empirical value of 135 .

The fatigue life distribution law of tunnel bottom structure under different structural types and the minimum logarithmic fatigue life of tunnel bottom structure under various working conditions are obtained by calculation, and the load cycle times of tunnel bottom structure under fatigue failure can be obtained. Combined with the operation parameters of heavy-haul railway, the calculation results of minimum fatigue logarithmic life under different invert risespan ratios are obtained, that is, the service life of tunnel structure.

Taking different invert rise-span ratios as examples, the service life of tunnel lining structure is calculated. According to the life calculation method, when the rise-span ratio is $1 / 8$, $1 / 10,1 / 12$, and $1 / 14$, the minimum logarithmic fatigue life of the tunnel is $6.879,6.728,6.631$, and 6.550 , respectively. It can be seen that the load cycles of the tunnel lining structure when the fatigue failure occurs are 106.879, 106.728, 106.631, and 106.550 , because a pair of unit 10000-ton train tunnels are considered in the fatigue calculation. In the case of single intersection, the service life is as follows:

$$
\begin{aligned}
& \text { 1/8: } T=\frac{10^{6.879}}{365 \times 135}=153(\text { years }), \\
& \text { 1/10: } T=\frac{10^{6.728}}{365 \times 135}=108 \text { (years), } \\
& \text { 1/12: } T=\frac{10^{6.631}}{365 \times 135}=86(\text { years }), \\
& \text { 1/14: } T=\frac{10^{6.550}}{365 \times 135}=72(\text { years }) .
\end{aligned}
$$

Similarly, the service life of tunnel lining structure under other different working conditions can be calculated in this way.

3.2. Transport Conditions. Consider that the total weight of the train is 32400 tons and the average daily operation number is 10 pairs. The fatigue life distribution law of tunnel bottom structure under different surrounding rock grade, invert (bedding) thickness, and rise-span ratio is obtained by calculation, and the minimum fatigue life of tunnel bottom structure under various working conditions, that is, the number of load cycles that the tunnel bottom structure bears when fatigue failure occurs, can be calculated by combining with the operation parameters of heavy-haul railway, and the reasonable value range of design parameters meets the service life.

3.3. Design Parameters of Tunnel Invert. Taking V-grade surrounding rock as an example, the service life of tunnel lining structure under different invert rise-span ratio conditions (the thickness of tunnel inverts is $20 \mathrm{~cm}$ and $30 \mathrm{~cm}$ ) can be calculated as in Table 7. The variation curve of service life with invert rise-span ratio is shown in Figure 3.

The following can be seen from Figure 3 and Table 7:

(1) Under the condition of grade $\mathrm{V}$ surrounding rock, when the invert thickness is $30 \mathrm{~cm}$, when the risespan ratio of tunnel invert is $1 / 5$, the fatigue life of 
TABLe 7: Tunnel service life (years) under different rise-span ratios.

\begin{tabular}{|c|c|c|c|c|}
\hline \multicolumn{5}{|c|}{ Rise-span ratio } \\
\hline Invert thickness & $1 / 8$ & $1 / 7$ & $1 / 6$ & $1 / 5$ \\
\hline $30 \mathrm{~cm}$ & 48 & 61 & 75 & 92 \\
\hline $20 \mathrm{~cm}$ & 36 & 43 & 53 & 65 \\
\hline
\end{tabular}

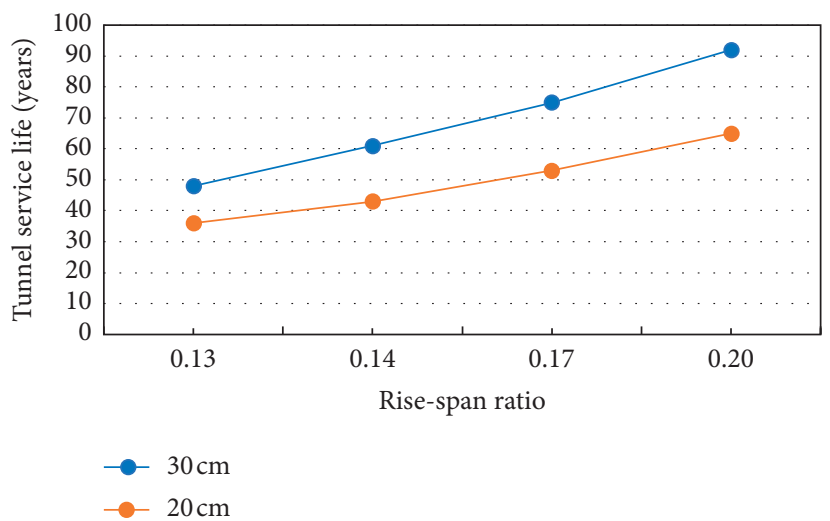

FIGURE 3: Relationship between tunnel structure life and invert rise-span ratio.

heavy-haul railway tunnel structure is 92 years; when the rise-span ratio is $1 / 6$, the fatigue life of tunnel structure is 75 years; when the rise-span ratio is $1 / 7$, the fatigue life of tunnel structure is 61 years, which meets the design service life (50 years); when the rise-span ratio is $1 / 8$, the tunnel structure fatigue life is poor. The service life is 48 years, which is less than the design service life (50 years), which cannot meet the requirements of structural antifatigue.

(2) Under the condition of V-grade surrounding rock and the invert thickness of $20 \mathrm{~cm}$, when the rise-span ratio of tunnel invert is $1 / 5$, the fatigue life of heavyhaul railway tunnel structure is 65 years. And when the rise-span ratio is $1 / 6$, the fatigue life of tunnel structure is 53 years, which meets the design service life (50 years); when the rise-span ratio is $1 / 7$ and $1 / 8$, the fatigue life of tunnel structure is 43 and 36 years, respectively. It is less than the design service life ( 50 years), which cannot meet the requirements of structural fatigue resistance.

(3) From the perspective of the relationship between the fatigue life of tunnel structure and the rise-span ratio of invert, the fatigue life also has a corresponding increasing trend with the increase of invert rise-span ratio. It shows that the fatigue resistance of heavyhaul railway tunnel structure can be significantly improved by optimizing the rise-span ratio of tunnel invert.

(4) According to the relationship curve between the fatigue life of tunnel structure and the rise-span ratio of invert, and based on the design service life of 50 years, it can be estimated that the thickness value of invert should be greater than or equal to $20 \mathrm{~cm}$; and the rise-span ratio value should be greater than or equal to $1 / 6$.

(5) Under the condition of IV-grade surrounding rock, it is suggested that the thickness value of invert should be greater than or equal to $15 \mathrm{~cm}$; and the rise-span ratio should be greater than or equal to $1 / 7$. Under the condition of III-grade surrounding rock, it is suggested that the thickness of invert should be greater than or equal to $10 \mathrm{~cm}$; and the rise-span ratio should be greater than or equal to $1 / 8$.

3.4. The Thickness of Tunnel Bottom. The service life of tunnel bottom structure with different thickness can be calculated as shown in Table 8 . The variation curve of service life with bedding thickness can be calculated as shown in Figure 4.

(1) When the thickness of tunnel bottom is $30 \mathrm{~cm}$, the service life of tunnel bottom structure is 89 years; and when the thickness of tunnel bottom is $20 \mathrm{~cm}$, the service life of tunnel bottom structure is 58 years, which can meet the design service life (50 years) of the 40-ton axle load heavy-haul railway tunnel; when the thickness of tunnel bottom is $10 \mathrm{~cm}$ and $15 \mathrm{~cm}$, the service life of tunnel bottom structure is 32 years and 47 years, respectively. It is less than the design service life (50 years), which cannot meet the requirements of structural antifatigue.

(2) According to the relationship curve between the fatigue life of tunnel structure and the thickness of pavement, and based on the design service life of a 40 -ton heavy-haul railway tunnel for 50 years, it can be estimated that the recommended bottom 
TABle 8: Service life of tunnel bottom structure with different bottom thickness.

\begin{tabular}{lllll}
\hline Thickness of bedding $(\mathrm{cm})$ & 10 & 15 & 20 & 30 \\
\hline Service life (years) & 32 & 47 & 58 & 89 \\
\hline
\end{tabular}

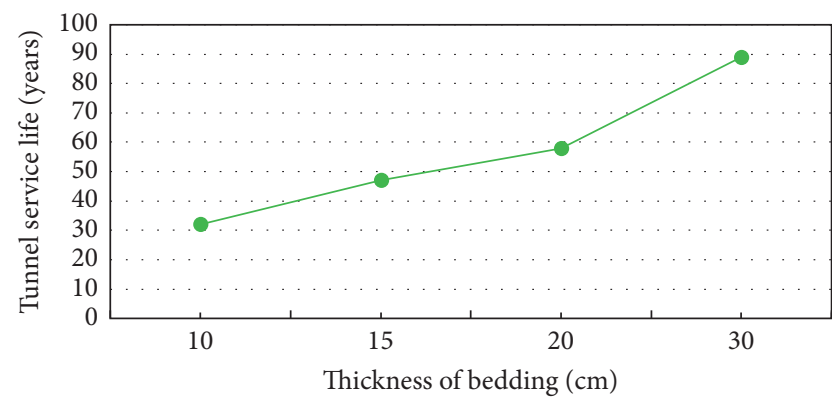

Figure 4: Relationship between service life of tunnel bottom structure and bottom thickness.

thickness should be no less than $20 \mathrm{~cm}$ under the condition of III-grade surrounding rock.

\section{Conclusion}

4.1. Dynamic Performance Analysis of Tunnel Bottom Structure. The dynamic response of heavy-haul railway tunnel foundation structure under different base structure design parameters is analyzed numerically. The main conclusions are as follows.

(1) The thicker invert is helpful to improve the stress state of the tunnel base structure. The larger the thickness of the invert, the smaller the vertical dynamic stress at the bottom of the invert, but the range of the dynamic stress changes with it, and the less the influence of train dynamic load on the surrounding rock, which indicates that the thicker inverted arch is beneficial to the long-term stress stability of the tunnel bottom stratum.

(2) Under the condition of large invert span ratio (1/5), the smaller the vertical dynamic stress at the bottom of tunnel invert is $(45.885 \mathrm{kPa})$, the better improved the internal force of inverted arch structure can be, which can reduce the requirements of structural thickness and reinforcement to a certain extent and improve the stability and safety of the structure. But on the other hand, a large rise-span ratio, corresponding to thick invert filling, will increase the project cost to a certain extent.

(3) The worse the surrounding rock grade is, the more obvious the vertical dynamic stress attenuation along the base structure is; the greater the invert thickness is, the lower the load transfer rate at the bottom of inverted arch is; and the greater the invert rise-span ratio is, the lower the load transfer rate at the bottom of inverted arch is.
4.2. Design Parameters of Heavy-Haul Railway Tunnel Bottom with 40-Ton Axle Load. According to the calculation and analysis of the dynamic response and fatigue life of the tunnel bottom structure under the above different tunnel design parameters, taking the tunnel design service life of 50 years as the benchmark, the tunnel bottom structure type and design parameters under different conditions can be determined as follows:

(1) Structural type: inverted arch shall be set in sections of III-grade and above. Whether an invert is set in sections of II-grade and below shall be determined according to lithology and groundwater conditions. When the surrounding rock condition is good and the tunnel bottom structure (without invert structure) is adopted, the thickness is recommended to be more than $20 \mathrm{~cm}$, and the concrete strength grade should not be lower than C35.

(2) Connection mode: the inverted arch and side wall of the tunnel should be connected in sequence to reduce the stress concentration and improve the stress state of the connection part between the inverted arch and the side wall.

(3) Rise-span ratio: when invert structure is adopted, considering the angle of ensuring the filling thickness and service life of the tunnel bottom, it is suggested that the rise-span ratio of invert of single-track tunnel should be $1 / 6 \sim 1 / 8$; the larger value should be taken when the surrounding rock condition is poor and the small value should be taken when the surrounding rock condition is good.

(4) Invert thickness: when inverted arch structure is adopted, the thickness of inverted arch is recommended to be no less than $20 \mathrm{~cm}$ under the condition of $\mathrm{V}$-grade surrounding rock, to be no less than $15 \mathrm{~cm}$ under IVgrade surrounding rock, and to be no less than $10 \mathrm{~cm}$ under the condition of III-grade surrounding rock and II-grade surrounding rock sections requiring inverted arch.

(5) Bottom thickness: according to the relationship curve between the service life of tunnel bottom structure and the thickness of pavement, taking the tunnel design service life of 50 years as the benchmark, it can be calculated that the recommended value of bedding thickness meeting the design service life is $20 \mathrm{~cm}$ under the condition of II-grade surrounding rock.

\section{Data Availability}

The data used to support the findings of this study are available within the article.

\section{Conflicts of Interest}

The authors declare that there are no conflicts of interest regarding the publication of this paper. 


\section{Acknowledgments}

Thanks are due to the support of the Scientific Research Fund of China Academy of Railway Sciences Group Co., Ltd. (no. 2018YJ030).

\section{References}

[1] J. Jakobsen, "Ground vibration from rail traffic," Journal of Low Frequency Noise, Vibration and Active Control, vol. 6, no. 3, pp. 96-103, 1987.

[2] T. M. Dawn and C. G. Stanworth, "Ground vibrations from passing trains," Journal of Sound and Vibration, vol. 66, no. 3 , pp. 355-362, 1979.

[3] W. H. Zou, M. Zhang, Y. Q. Liu, and W. B. Ma, "Stress distribution and dynamic response of foundation structure of heavy haul railway tunnel under 30t axle load," China Railway Science, vol. 37, no. 5, pp. 50-57, 2016.

[4] Z. Q. Li, Q. Wu, and Y. M. Cheng, Study on Dynamic Characteristics and Design Method of Heavy Haul Railway Tunnel Structure, Southwest Jiaotong University, Changsha, China, 2018.

[5] W. H. Chang, Study on Mechanical Characteristics of Heavy Haul Railway Track Structure with 30t Axle Load, Beijing Jiaotong University, Beijing, China, 2009.

[6] J. X. Huo, "Disease characteristics and fatigue damage mechanism of heavy haul railway tunnel bottom structure," Railway Construction, vol. 59, no. 7, pp. 61-64, 2019.

[7] C. Li, L. M. Peng, M. F. Lei, and N. Liu, "Experimental study on fatigue performance and cumulative damage of substructure of heavy haul railway tunnel," China Railway Science, vol. 40, no. 4, pp. 77-85, 2019.

[8] H. R. Song, Structural Parameter Dynamic Analysis of Ballastless Track in Heavy Haul Railway Tunnel Section, Southwest Jiaotong University, Changsha, China, 2015.

[9] D. Y. Li and L. N. Y. Wong, "The Brazilian disc test for rock mechanics applications: review and new insights," Rock Mechanics And Rock Engineering, vol. 46, no. 2, pp. 269-287, 2018.

[10] D. Y. Li, Q. Q. Zhu, Z. L. Zhou, X. B. Li, and P. G. Ranjith, "Fracture analysis of marble specimens with a hole under uniaxial compression by digital image correlation," Engineering Fracture Mechanics, vol. 183, pp. 109-124, 2017.

[11] D. Li, Z. Sun, T. Xie, X. Li, and P. G. Ranjith, "Energy evolution characteristics of hard rock during triaxial failure with different loading and unloading paths," Engineering Geology, vol. 228, pp. 270-281, 2017.

[12] Z. Z. Wang and Z. Zhang, "Seismic damage classification and risk assessment of mountain tunnels with a validation for the 2008 Wenchuan earthquake," Soil Dynamics and Earthquake Engineering, vol. 45, pp. 45-55, 2013.

[13] J. F. Chai, A. B. Jin, Y. T. Gao et al., "Water inrush inoculation process in mines based on moment tensor inversion," Chinese Journal of Engineering, vol. 37, no. 3, p. 267, 2015.

[14] Z. Z. Wang, L. Jiang, and Y. Gao, "Shaking table test of seismic response of immersed tunnels under effect of water," Soil Dynamics and Earthquake Engineering, vol. 116, pp. 436-445, 2019.

[15] Z. Z. Wang and C. Chen, "Fuzzy comprehensive Bayesian network-based safety risk assessment for metro construction projects," Tunnelling and Underground Space Technology, vol. 70, pp. 330-342, 2017.

[16] Z. Z. Wang, Y.-J. Jiang, C. A. Zhu, and T. C. Sun, "Shaking table tests of tunnel linings in progressive states of damage,"
Tunnelling and Underground Space Technology, vol. 50, pp. 109-117, 2015.

[17] J. F. Chai, A. B. Jin, and S. C. Wu, "Rock fracture mechanism and its evolution law of triaxial compression test based on P-T distribution diagram method," Electronic Journal of Geotechnical Engineering, vol. 20, no. 28, pp. 13451-13464, 2015.

[18] J. F. Chai, A. B. Jin, and S. C. Wu, "Mechanism and evolution law of rock failure based on moment tensor inversion," Electronic Journal of Geotechnical Engineering, vol. 20, no. 15, pp. 6659-6672, 2015.

[19] J. F. Chai, Y. T. Gao, S. C. Wu et al., "Research on single jointed rock failure mechanism based on mesoscopic characteristics analysis," Journal of Mining and Safety Engineering, vol. 33, no. 3, pp. 528-534, 2016.

[20] F. Liu and Y. Cheng, "The improved element-free Galerkin method based on the nonsingular weight functions for inhomogeneous swelling of polymer gels," International Journal of Applied Mechanics, vol. 10, no. 04, p. 1850047, 2018.

[21] F. Liu, Q. Wu, and Y. Cheng, "A meshless method based on the nonsingular weight functions for elastoplastic large deformation problems," International Journal of Applied Mechanics, vol. 11, no. 01, Article ID 1950006, 2019.

[22] Q. Wu, F. B. Liu, and Y. M. Cheng, "The interpolating element-free Galerkin method for three-dimensional elastoplasticity problems," Engineering Analysis with Boundary Elements, vol. 115, pp. 156-167, 2020.

[23] S. Y. Yu, M. J. Peng, H. Cheng, and Y. M. Cheng, "The improved element-free Galerkin method for three-dimensional elastoplasticity problems," Engineering Analysis with Boundary Elements, vol. 104, pp. 215-224, 2019. 